\title{
Left ventricular dysfunction in the fetus: relation to aortic valve anomalies and endocardial fibroelastosis
}

\author{
Gurleen K Sharland, Sunder K Chita, Nuala L K Fagg, Robert H Anderson, \\ Michael Tynan, Andrew C Cook, Lindsey D Allan
}

\begin{abstract}
Objective-To examine the relation between a characteristic form of left ventricular dysfunction in the fetus and abnormalities of the aortic valve and endocardial fibroelastosis of the left ventricle.
\end{abstract}

Design-A retrospective study to examine the correlation between echocardiographic findings in the fetus and postnatal or necropsy findings.

Setting-Tertiary referral centre for fetal echocardiography.

Patients-Thirty fetuses showing a characteristic echocardiographic picture of left ventricular dysfunction.

Main outcome measures-The relation between the prenatal echocardiographic features and the postnatal and necropsy findings.

Results-At presentation the size of the left ventricular cavity was normal or enlarged in all cases. The measurements of the orifice of the aortic root and mitral valve were either normal or small for the gestational age. The echocardiographic diagnosis made at presentation was critical aortic stenosis in all cases. At necropsy or postnatal examination the aortic valve was dysplastic and stenotic in 15 cases and the left ventricle had become hypoplastic in one of these. Aortic atresia was present in seven patients, three of whom had a hypoplastic left ventricle. In six patients the aortic valve was bicuspid although not obstructive. One of these patients had hypoplasia of the aortic arch and one had a hypoplastic left ventricle but in the remaining four patients endocardial fibroelastosis of the left ventricle was the only abnormality found. No follow up information was available in two. Of 26 patients for whom there was postmortem information, 24 had evidence of some degree of endocardial fibroelastosis of the left ventricle. Sequential observations showed that five cases developed into the hypoplastic left heart syndrome.

Conclusions-This type of left ventricular dysfunction in the fetus is the result of an overlap of diseases, including primary left ventricular endocardial fibroelastosis, critical aortic stenosis, and the hypoplastic left heart syndrome.
Over the past 10 years echocardiography has become established as a reliable technique for the detection of structural heart disease in prenatal life. ${ }^{12}$ As the number of anomalies detected increases it becomes possible to examine groups of cases in separate diagnostic categories. The characteristic echocardiographic and anatomical features of the defect as encountered in the fetus can then be described, while the severity and outcome of the lesion seen antenatally can be compared with postnatal experience. The natural history and progression of lesions can be observed from the second trimester. With these aims we retrospectively examined a series of 30 cases seen over a 10 year period in which there were the characteristic echocardiographic features of left ventricular dysfunction.

Patients and methods

The case material was derived from patients referred for fetal echocardiography to our unit since February 1980 . We now have experience of 7000 pregnancies at increased risk of congenital heart disease. ${ }^{3}$ Between 1980 and 1983 we evaluated the fetal heart with cross sectional imaging only on an Advanced Technical Laboratories Mark 3 mechanical sector scanner. Later, Doppler evaluation was added to the examination. Since 1985, an Advanced Technical Laboratories Ultramark 4 and a Hewlett-Packard 77020 phased array imaging system have been used. The $5 \mathrm{MHz}$ transducer was used routinely but a $3.5 \mathrm{MHz}$ transducer was also available. Colour flow mapping became available in 1987. The gestational age at the time of examination was between 18 and 40 weeks and was confirmed by measuring the femoral length or biparietal diameter.

Of 462 cases of detected structural heart malformation, 30 were considered to have similar echocardiographic features affecting the left ventricle at presentation. The diagnostic criteria for inclusion in the group were a poorly contracting left ventricle where there was no measurable difference in the dimensions of the cavity between systole and diastole and a left ventricular cavity size where either the maximum width or length was normal or increased for the gestational age. All the cases included in this study were initially diagnosed as having critical aortic stenosis. No other cases of critical aortic stenosis have been excluded. During the same time period 77 cases were diagnosed at presentation as hypoplastic left 
heart syndrome and these were excluded from this study. In another three patients there was evidence of left ventricular dysfunction but there was also right ventricular dysfunction and the aorta seemed normal. These were excluded from this study because they were diagnosed as having a different disease both on prenatal echocardiographic examination and on subsequent examination after delivery. There was one further fetus, with an aortic-left ventricular tunnel, that also had evidence of left ventricular dysfunction. However, there was considerable aortic incompetence at presentation which distinguished this case from the study group. All cases of isolated left ventricular endocardial fibroelastosis were included in this study. There were five cases of endocardial fibroelastosis involving more than one cardiac chamber; these were excluded from the study because they did not have the features of critical aortic stenosis and were considered to belong in a different diagnostic group at presentation.

In 28 of the 30 cases forming the study group the patient was referred because of the suspicion of fetal heart disease on a routine obstetric scan. The reason for referral in one case was cystic hygroma and this was the only fetus in which an extracardiac anomaly was found. Fetal hydrops was the reason for referral in the last case.

A videotape recording of each study was reviewed retrospectively and the echocardiographic findings noted. Evidence of fetal hydrops, as shown by fetal skin oedema, ascites, and pleural or pericardial effusions, was sought and recorded. The orifices of the mitral valve and the aortic root and the length and maximum width of the left ventricle were measured and compared with the normal values for the gestational age. ${ }^{45}$ The aortic valve was qualitatively examined and graded as being inadequately visualised, normal, or

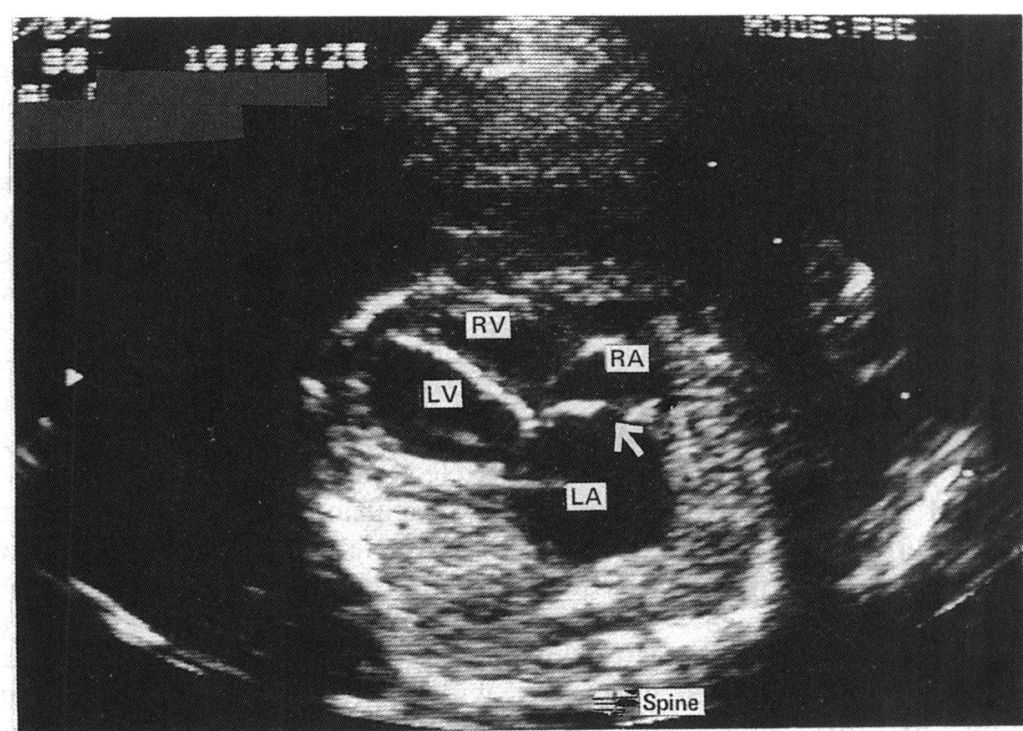

Figure 1 Four chamber echocardiogram of the heart of a typical case. The overall size of the heart is increased. The left ventricle appears larger than the right and the left atrium is enlarged. The walls of the left ventricle show increased echogenicity compared with the right ventricle. The arrow indicates the atrial septum which is restricted. The walls of the left ventricle show increased echogenicity. $L V$, left ventricle; $R V$, right ventricle; $L A$, left atrium; $R A$, right atrium.

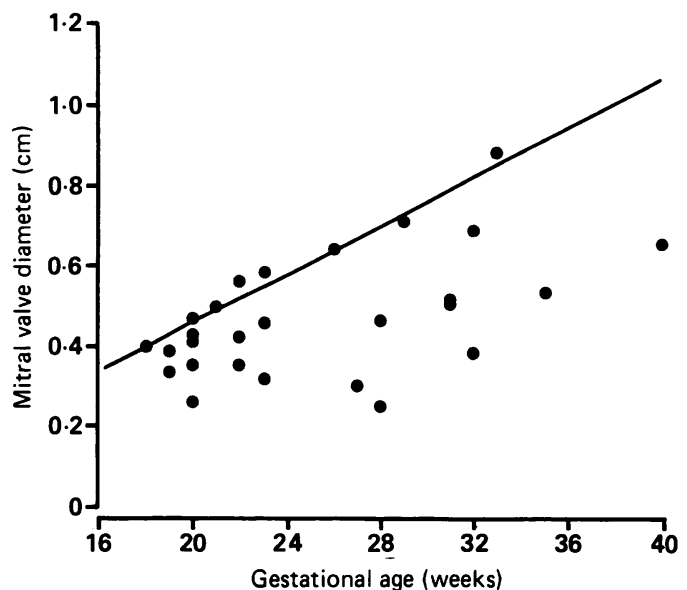

Figure 2 Distribution of mitral valve orifice size in 27 of the fetuses in relation to gestational age. The values are compared with the mean of the normal range for gestational age (solid line). For the mean, $M V=-0.146+0.03$ gestational age.

thickened. The sizes of the left and right atria were compared and the left atrial size was graded as normal and as mildly or moderately enlarged relative to the right atrium. The cardiothoracic ratio was measured on a four chamber view in a standard fashion and compared with the normal range. ${ }^{6}$ The Doppler characteristics of flow of blood across the mitral and aortic valves recorded on videotape were noted. Five patients had sequential studies during pregnancy. The necropsy specimens of 21 cases were available for examination and the postmortem reports of a further five cases were studied. In 16 cases the specimens were available for postmortem examination soon after the echocardiographic study. In these we calculated the agreement between the measurements made echocardiographically and those made at necropsy. ${ }^{7}$ In four cases necropsy was refused but in two of these postnatal echocardiography had been performed. No follow up information was available in two cases. The clinical features and outcome of each case were analysed.

\section{Results}

There was evidence of fetal hydrops at presentation in a total of nine cases.

\section{ECHOCARDIOGRAPHIC FEATURES}

Mitral valve, aortic valve, left ventricle

The characteristic echocardiographic features are best seen on a four chamber view (fig 1 ). The mitral valve orifice and the aortic root size were normal or small for the gestational age and figures 2 and 3 show the scatter of values compared with the mean of the normal range. The excursion of the leaflets of the mitral valve was limited in all cases. Direct examination of the aortic valve was inadequate in 11 cases but thickened leaflets were seen in 13 of the remaining cases. The quality of images depended on adequate visualisation, whatever the gestational age of the subject; thickened leaflets were more convincingly identifiable in later pregnancy. The maximum left ventricular 
Figure 3 Distribution of measured in 29 of the fetuses and plotted against gestational age. The values in two fetuses were the same. The values are compared with the mean of the normal range for gestational age (solid $A o=-0.138+0.0224$ gestational age. the aortic root size line). For the mean,

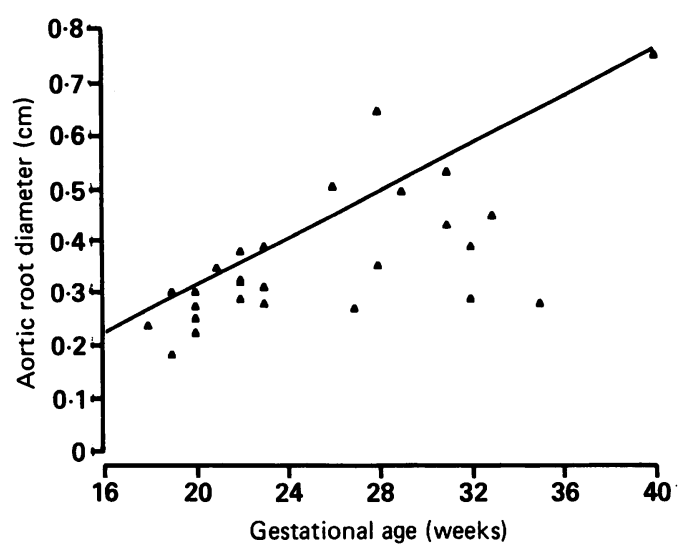

width was either within the normal range or increased compared with the normal range in all cases (fig 4A); the length was normal or increased in most (fig 4B).

\section{Atrial septum, left atrial size, and cardiothoracic} ratio

The left atrium was normal in 18, slightly enlarged in five, and moderately enlarged in seven. The cardiothoracic ratio, which varied, depended mainly on the left atrial size and the presence or absence of fetal hydrops (fig 5). The ratio was increased in all hydropic fetuses and in all those with a large left atrium and associated intact atrial septum. The atrial septum seemed to be intact in 11 cases, with the septum bulging from left to right. In all these cases the left atrium was enlarged. In six cases left to right shunting through the atrial septum was clearly shown on colour Doppler echocardiography. In these cases the flap valve of the foramen ovale, normally a prominent mid-left atrial structure, was seen herniating into the right atrium.

\footnotetext{
Figure 4- (A) Maximum left ventricular width measured on a four chamber view in 29 fetuses and plotted against the gestational age on a graph showing the normal range for this variable. For the mean of the normal range, mean of the normal range, 0.043839 gestational age. (B) Maximum left ventricular length measured on a four measured on a four
chamber view in 29 fetuses and plotted against the gestational age values in two fetuses were the same. Mean of the normal range, MV length $=0.40822+$ $L V$ length $=0.40822+$
0.080163 gestational age.
}
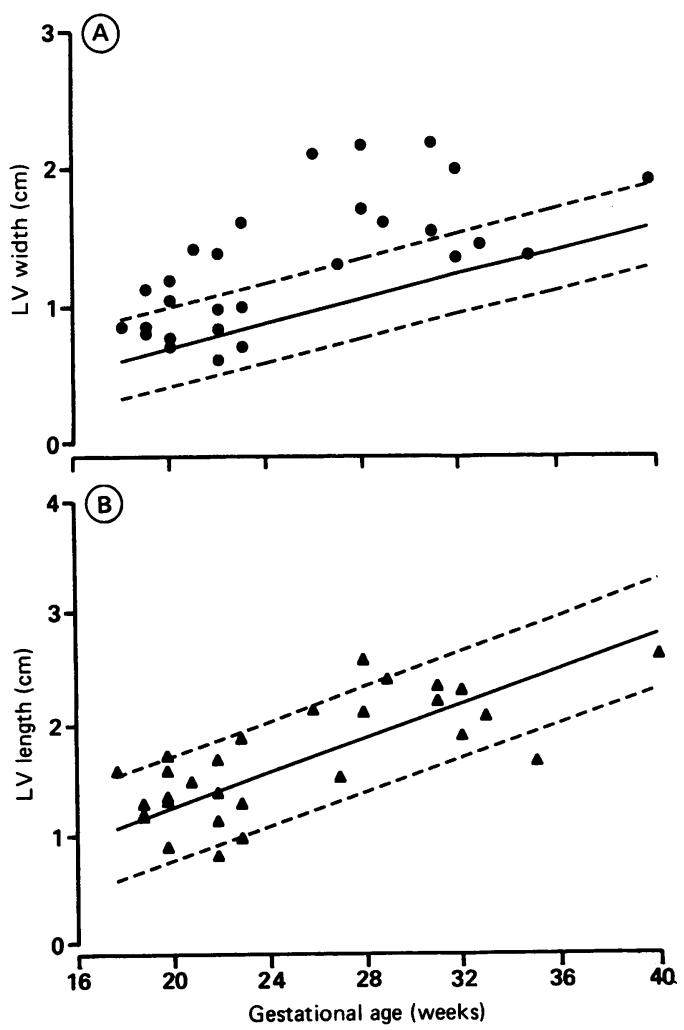

Left ventricular echogenicity

In 28 cases the left ventricle was more echogenic than the right ventricle-in 14 to a considerable extent.

Doppler echocardiography

In 18 of the 22 cases that were examined by pulsed or colour Doppler echocardiography there was no forward flow into the left ventricle. Mitral regurgitation was detected in six cases. Forward flow was demonstrated in the aorta in 15 cases; in five of them the velocity was greater than the normal values for gestational age. ${ }^{58}$ In six cases reverse flow only could be demonstrated in the aorta and in one case no flow was detectable. In three cases there was also tricuspid regurgitation.

\section{SEQUENTIAL STUDIES}

Five patients had sequential studies: at 31 and 36 weeks, 22 and 28 weeks, 22 and 32 weeks, 32 and 34 weeks, and 31 and 41 weeks respectively. The measurements of the mitral orifice and aortic root tended to remain static or grow at a slower rate than expected as pregnancy advanced, with the result that the values became smaller compared with the normal mean for gestational age. Similarly, sequential measurements of the left ventricular cavity showed either no growth or a reduced rate of growth so that with advancing gestational age the left ventricle became progressively smaller compared with the mean for gestation and also with the size of the right ventricle (figs $6 \mathrm{~A}$ and $B)$. In one case the cavity size was initially very large and although the left ventricle remained large, the rate of growth was reduced so that the ratio of the left to the right ventricle decreased with advancing gestational age. All five cases had evidence of forward flow across the aortic valve on Doppler echocardiography at presentation. In two cases, however, no forward flow could be demonstrated on the subsequent study. Both of these cases progressed to hypoplastic left heart syndrome. In one of these, hypoplastic left heart was diagnosed on the

$$
\begin{aligned}
& \Delta \text { Intact atrial septum } \\
& \text { - Hydropic } \\
& \text { a Non-hydropic, patent foramen }
\end{aligned}
$$

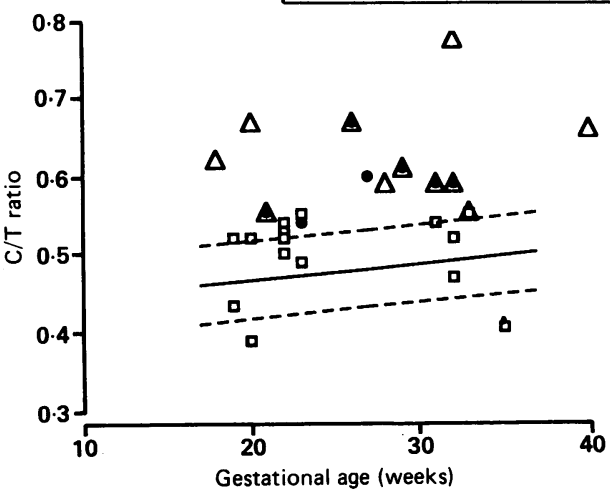

Figure 5 Cardiothoracic $(C / T)$ ratio in the study group $n=30)$ compared with the normal range for gestational age. Cases that were hydropic and had an intact septum are indicated by a solid circle within the triangle. The values for two hydropic fetuses and one non-hydropic fetus overlapped. 
Figure 6 (A) Sequential measurements of left ventricular width in five cases compared with the normal range for

advancing gestational age. (B) Sequential left ventricular length measurements in five cases compared with the normal range for advancing gestational age.
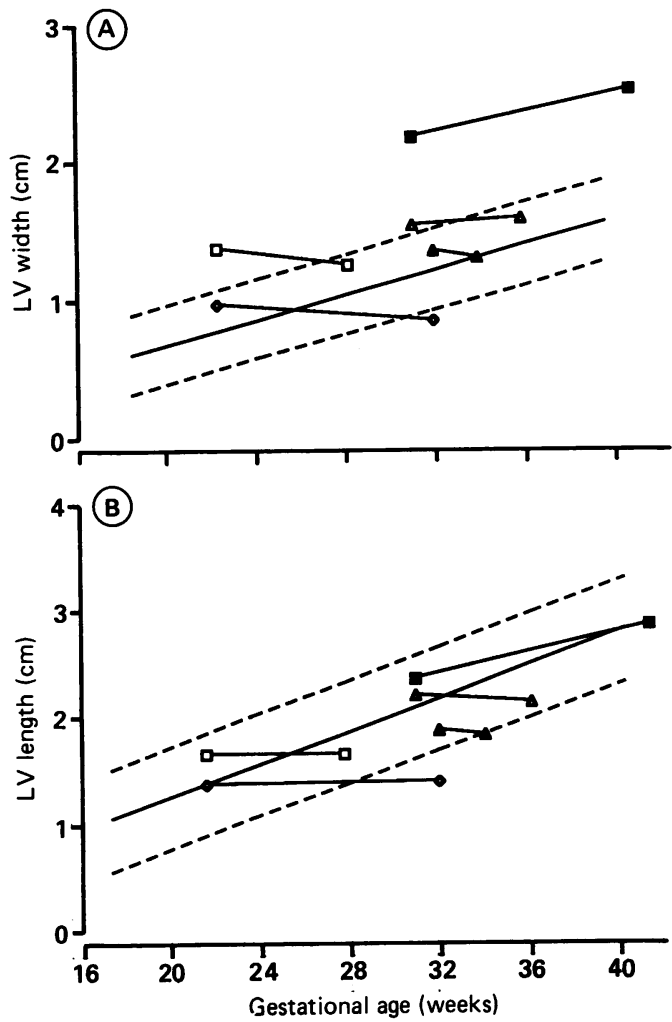

sequential echocardiographic study and in the second the diagnosis was made at postmortem study after intrauterine death a few weeks after the sequential study. The atrial septum in this case, which was first seen at 22 weeks' gestation, was initially patent whereas at 28 weeks' gestation it appeared to be intact by echocardiography. This was confirmed at necropsy. In addition, in a further three fetuses that only had one prenatal study, necropsy three, four, and eight weeks after the echocardiographic study showed that the left ventricle was hypoplastic relative to the right ventricle and hypoplastic left heart syndrome was the final diagnosis.

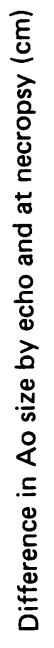

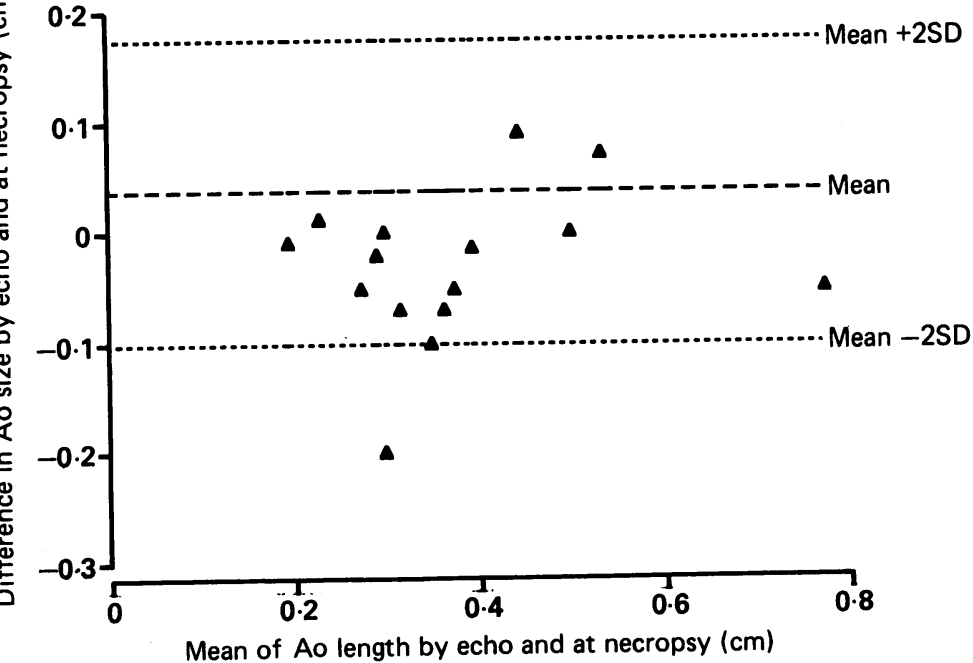

Figure 7 Agreement (differences versus mean) between aortic root measurements made echocardiographically and in the same fetuses at necropsy. Only fetuses in which the interval between the two examinations was $<$ two weeks are included $(n=16$, mean of differences $=0.035, S D$ of differences $=0.069)$.
OUTCOME

Eighteen mothers presented before 24 weeks' gestation and 16 of these opted for termination of pregnancy. There were two spontaneous intrauterine deaths. Twelve patients were born alive, four of whom were in cardiac failure at delivery. All but one died within the first week of life, six on the first day. One neonate who had had a balloon dilatation of the aortic valve prenatally died at 37 days. ${ }^{9}$ Postnatal balloon dilatation of a valve was performed in six cases but despite successful relief of valve obstruction the left ventricular output was inadequate to maintain the circulation.

\section{POSTMORTEM AND POSTNATAL FINDINGS}

Left ventricle and aorta

The diagnosis of left ventricular disease was confirmed in each case where a specimen was available for examination. The left ventricular cavity and aorta of each specimen were measured. Sixteen specimens were available for postmortem examination soon after the echocardiographic study and in these there was good agreement between the echocardiographic and anatomical measurements. Figure 7 shows the agreement between the measurements for the aortic root and fig 8 that for the left ventricular length. In three cases, where there was an interval of three, four, and eight weeks between the echocardiographic and postmortem examinations, the left ventricular and aortic measurements were more suggestive of the diagnosis of the hypoplastic left heart syndrome.

Left ventricular endocardial fibroelastosis

The left ventricle of each available specimen was inspected for evidence of fibroelastosis (fig 9). In two cases in which the left ventricle was very echogenic there was only mild fibroelastosis in the specimen. In one case with severe fibroelastosis at necropsy there had only been slight echogenicity. In the remaining specimens the correlation between the two examinations was good. In 24 of the 26 specimens examined at necropsy there was some degree of endocardial fibroelastosis of the left ventricle.

\section{Atrial septum}

The atrial septum was found to be intact in five cases, all of which had been suspected on echocardiography. In a further four cases, the atrial septum was restrictive, three of which were thought to be intact echocardiographically. One case was thought to be intact echocardiographically but found to be patent at necropsy. A postmortem examination had not been performed in the two remaining cases in which an intact atrial septum was suspected.

\section{Final diagnosis}

Fifteen of 28 cases in whom a postnatal or postmortem study was performed had a dysplastic and stenotic aortic valve (fig 9); in one of them a hypoplastic left ventricle developed. Seven cases had aortic atresia; in three of them a hypoplastic left ventricle developed. In five of these seven forward flow was detected pre- 


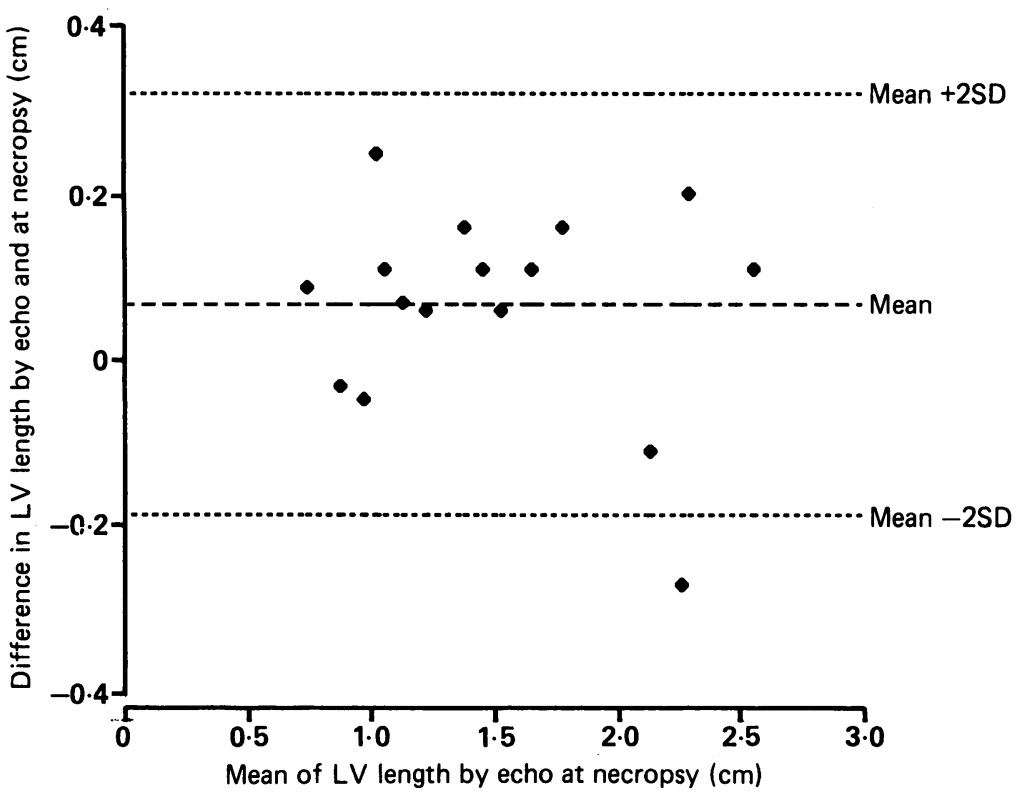

Figure 8 Agreement (differences versus mean) between left ventricular length measurements made echocardiographically and at necropsy in the same fetuses. ' Only fetuses in which the interval between the two examinations was $<$ two weeks are included $(n=16$, mean of differences $=0 \cdot 065, S D$ of differences $=0 \cdot 127)$.

natally across the aortic valve. In six cases the aortic valve was bicuspid although not obstructive. One of these had hypoplasia of the aortic arch and one developed a hypoplastic left ventricle, but endocardial fibroelastosis of the left ventricle was the only pathological finding in the remaining four cases. Thus of those with follow up information five cases had progressed to the hypoplastic left heart syndrome.

\section{Discussion}

We originally designated this characteristic echocardiographic picture of left ventricular dysfunction as critical aortic stenosis. There were six cases, however, that were indistinguishable echocardiographically from the rest but which proved to have a thin and patent aortic valve at necropsy. There were five cases that had a patent aortic valve on prenatal Doppler echocardiographic studies but in which the valve had become atretic by the time of necropsy. Development into the hypoplastic left heart syndrome was seen in five cases, as the left-sided structures failed to grow at a normal rate with advancing gestational age. This type

Figure 9 View of the open left ventricle of one of the cases at necropsy. The left ventricular cavity is dilated and there is considerable endocardial fibroelastosis lining the cavity of the ventricle. The aortic valve is thickened and dysplastic. AoV, aortic valve; $L V$, left ventricle; $M V$, mitral valve. of left ventricular dysfunction in the fetus, therefore, represents an overlap of diseases including primary left ventricular endocardial fibroelastosis, critical stenosis of the aortic valve, and the hypoplastic left heart syndrome. It constitutes over $6 \%$ of the abnormalities encountered in prenatal life compared with a frequency of $1.2 \%$ of critical aortic stenosis in a postnatal series. ${ }^{10}$ This discrepancy is accounted for by the observation that some of these cases seen in early pregnancy will progress to either aortic atresia or the hypoplastic left heart syndrome and that some cases have primary left ventricular disease and endocardial fibroelastosis.

Though the universal echocardiographic feature of this condition was a poorly contracting left ventricle, there were varied associated echocardiographic and anatomical findings. Left ventricular size was within the normal range for gestational age at presentation, but the size of the aortic root and mitral orifice was either normal or small for the gestational age. The echocardiographic diagnosis of endocardial fibroelastosis correlated well with the findings post mortem. Endocardial fibroelastosis was a frequent but not constant association. It also varied in extent and severity. Echocardiographically it proved impossible accurately to predict the morphology of the aortic valve in all the cases. If the aortic valve was well imaged, however, a thickened doming valve could be identified in some cases. At necropsy the leaflets of the aortic valve were commonly seen to be thickened and disorganised but in six cases they had a relatively normal appearance. The difficulty in comparing the echocardiographic and anatomical results is that in some cases there was an interval between the two and, as we found, changes can take place with advancing gestation.

Intrauterine closure of the atrial septum has been suggested as a cause of malformations of the left heart ${ }^{11}$ and a closed or restrictive atrial septum was confirmed at necropsy in eight cases. In one case seen at 22 and 28 weeks' gestation, the septum appeared patent at the first scan and closed six weeks later. It therefore seems possible that in those with an intact septum the flap was held shut by the raised left atrial pressure and became adherent as a secondary phenomenon. In five cases, furthermore, the left atrial pressure was forcing the flap valve into the right atrium and left to right flow was seen on colour flow mapping. Thus an intact atrial septum may be a consequence rather than a cause of the lesions in the left heart.

It is commonly assumed that cardiac malformation is well tolerated by the fetus. There was evidence of intrauterine cardiac failure, none the less, at the initial examination in nine cases. One of these fetuses died in utero. The four who reached term died on the first day of life. All 12 babies that were born alive had a normal vaginal delivery but were in a poor condition from birth. It is possible that caesarean section might have resulted in a neonate in better condition for valvotomy or balloon dilatation of the mitral valve and this has been previously suggested. ${ }^{12}{ }^{13}$ But, there is a poor long term 
prognosis for this condition despite surgery ${ }^{14}$ and this has to influence the obstetric management. It is likely that those cases with severe fibrotic changes or a small left ventricle in addition to small mitral and aortic orifices, as seen in many of our cases, will not survive relief of the obstruction ${ }^{15} 16$ and therefore these cases perhaps should continue to be delivered normally.

Our observations have shown that some of these cases will develop into the hypoplastic left heart syndrome. This has important implications for many aspects of fetal echocardiography. The value of screening of the four chamber view of the heart during the routine obstetric scan at 18 weeks' gestation is well established in the United Kingdom. The results of this study suggest that some forms of severe left heart disease may not be fully developed at 18 weeks and therefore may be overlooked in the screening programe involving scanning at this time. Also, the progressive changes that may occur as pregnancy advances make the accurate prediction of prognosis in early pregnancy difficult. Thirdly, the outcome of this form of disease of the left heart in the fetus is poor so that various therapeutic options should be considered during fetal life. The diagnosis was made early enough for termination to be a possible option in 18 cases. Sixteen mothers $(89 \%)$ chose this course. Alternatively, relief of aortic valve obstruction during pregnancy might allow the left ventricle time for recovery and improvement in growth before birth and may prevent or even reverse the development of endocardial fibroelastosis. ${ }^{9}$ However, there may be problems with prenatal selection of patients because it can sometimes be difficult to be sure that the aortic valve is abnormal. But even with accurate selection, by the time the patient comes to our attention it may be too late in gestation to prevent endocardial fibroelastosis and left ventricular damage.

In summary, left heart dysfunction can be identified in utero from echocardiographic characteristics. The natural history in cases seen in early gestation seemed to be an evolution into the hypoplastic left heart syndrome. The outlook for those in our series seen in later pregnancy has been universally bleak. These observations allow a more rational approach to possible treatment. The options include termination of pregnancy; the exploration of methods of changing the course of the disease process during gestation; planning the delivery to obtain the best possible neonatal condition; and preparation for immediate valvotomy in selected infants.

GKS, SKC, ACC, RHA, and LDA are supported by the British Heart Foundation. MT is supported by the Joseph Levy Foundation.

1 Kleinman CS, Hobbins JC, Jaffe CC, Lynch DC, Talner NS. Echocardiographic studies of the human fetus: prenatal diagnosis of congenital heart disease and cardiac dysrhythmias. Pediatrics 1980;65:1059-68.

2 Allan LD, Crawford DC, Anderson RH, Tynan MJ. Echocardiographic and anatomical correlations in fetal congenital heart disease. Br Heart J 1984;52:542-8.

3 Sharland G, Allan L. Detection of congenital abnormalities of the cardiovascular system by ultrasound. In: Chamberlain G, ed. Modern antenatal care of the fetus. Oxford: Blackwell Scientific Publications, 1990:356.

4 Allan LD. Manual of fetal echocardiography. Lancaster: MTP Press, 1986:52,65,70.

5 Allan LD, Chita SK, Al-Ghazali W, Crawford DC, Tynan M. Doppler echocardiographic evaluation of the normal $M$. Doppler echocardiographic evaluation of the
human fetal heart. Br Heart $J$ 1987;57:528-33.

6 Palladini D, Chita SK, Allan LD. Prenatal measurement of the cardiothoracic ratio in the evaluation of heart disease. Arch Dis Child 1990;65:20-3.

7 Bland JM, Altman DG. Statistical methods for assessing agreement between two methods of clinical measurement. Lancet 1986;i:307-10.

8 Kenny JF, Plappert $T$, Doubilet $P$, et al. Changes in intracardiac blood flow velocities and right and left ventricular stroke volumes with gestational age in the normal human fetus: a prospective Doppler echocardiographic study. Circulation 1986;74:1208-16.

9 Maxwell D, Allan L, Tynan MJ. Balloon dilatation of the aortic valve in the fetus: a report of two cases. Br Heart $J$ 1991;65:256-8.

10 Fyler DC, Buckley LP, Hellenbrand WE, Cohn HE. Report of the New England Regional Infant Cardiac Care Program. Pediatrics 1980;65(suppl):376.

11 Atkins DL, Clark EB, Marvin WJ. Foramen ovale/atrial septum ratio: a marker or transatrial blood flow. Circulation 1982;66:281-3.

12 Huhta JC, Carpenter RJ, Moise KJ, Deter RL, Ott DA, McNamara DG. Prenatal diagnosis and postnatal McNamara DG. Prenatal diagnosis and postnatal management of

13 Robertson MA, Byrne PJ, Penkoske PA. Perinatal management of critical aortic valve stenosis diagnosed by fetal echocardiography. Br Heart J 1989;61:365-7.

14 Zeevi B, Keane JF, Castenada AR, Perry SB, Lock JE. Neonatal critical valvar aortic stenosis: a comparison of surgical and balloon dilation therapy. Circulation 1989;80:831-9.

15 Latson LA, Cheatham JP, Gutgesell HP. Relation of the echocardiographic estimate of left ventricular size to mortality in infants with severe left ventricular outflow obstruction. Am J Cardiol 1981;48:887-91.

16 Rhodes LA, Colan SD, Sanders SP. Predictors of survival in neonates with critical aortic stenosis [abstract]. Circulation 1989;80 (suppl II): 186 